\section{Fatores associados ao tempo de espera e ao acesso ao transplante renal em Belo Horizonte, Minas Gerais, Brasil}

\author{
Factors associated with waiting time and access to \\ kidney transplants in Belo Horizonte, Minas Gerais \\ State, Brazil'
}

\author{
${ }^{1}$ Faculdade de Medicina, \\ Universidade Federal de \\ Minas Gerais, Belo Horizonte, \\ Brasil. \\ 2 Instituto de Ciências Exatas, \\ Universidade Federal de \\ Minas Gerais, Belo Horizonte, \\ Brasil. \\ 3 Gerência de Epidemiologia \\ e Informação, Secretaria \\ Municipal de Saúde de Belo \\ Horizonte, Belo Horizonte, \\ Brasil. \\ Correspondência \\ E. L. Machado \\ Departamento de Medicina \\ Preventiva e Social, \\ Faculdade de Medicina, \\ Universidade Federal de \\ Minas Gerais. \\ Av. Prof. Alfredo Balena 190, \\ Belo Horizonte, $M G$ \\ 30130-100, Brasil. \\ elainemachado77@yahoo. \\ com.br
}

\begin{abstract}
The objective of this study was to analyze factors associated with access to kidney transplants from living and cadaver donors in Belo Horizonte, Minas Gerais State, Brazil. The authors conducted a non-concurrent cohort study of patients on the waiting list for kidney transplants from 2000 to 2004 and followed until transplantation, death, exclusion, or continued presence on the line at the end of the study on December 31, 2005. The Cox model was used for competing risks. Of the 835 patients, 22.7\% were transplanted. Lower risk of transplantation from living donors and cadavers was observed in patients with more time on dialysis and blood type O. Lower risk of transplantation from living donors was observed in residents in a high health risk area and in recipients with diabetes. The greatest disparity in access was observed in transplants from living donors, since there were no significant socio-demographic differences in transplants from cadaver donors. One can infer that the organ allocation system contributed to mitigating socio-demographic inequalities, and that clinical issues were more relevant in access to transplants from cadaver donors.
\end{abstract}

Chronic Renal Insufficiency; Kidney Transplantation; Health Services Accessibility
Elaine Leandro Machado 1

Isabel Cristina Gomes 2

Francisco de Assis Acurcio 1

Cibele Comini César 2

Maria Cristina de Mattos Almeida ${ }^{3}$

Mariangela Leal Cherchiglia ${ }^{1}$

\section{Introdução}

A doença renal crônica em sua fase terminal representa um importante problema de saúde pública por ser uma condição extremamente delibilitante, associada com significativa morbidade, mortalidade e elevado custo de seu tratamento 1,2 . O portador dessa doença somente sobrevive com a utilização de métodos de filtragem artificial do sangue, as diálises (diálise peritoneal ou hemodiálise), ou a realização do transplante renal, as denominadas terapias de substituição renal. Além do elevado custo econômico, devem-se considerar as repercussões sociais, psicológicas e de restrição de qualidade de vida do paciente submetido às terapias de substituição renal ${ }^{3}$. Para pacientes elegíveis, o transplante renal representa um tratamento durável com benefícios na sobrevida, qualidade de vida e custo-efetividade, quando comparado às diálises 4,5,6.

Atualmente, o Brasil possui o maior sistema público de transplantes do mundo, subsidiando 95\% desse tratamento, incluindo procedimento cirúrgico, medicação e acompanhamento necessários ao pós-transplante 7 . Para que essa intervenção seja feita, são necessárias avaliações médica, cirúrgica e psicossocial do paciente, além da realização de exames e verificação da compatibilidade do possível doador em relação aos antígenos leucocitários humanos (HLA) ${ }^{8}$. Os transplantes renais são realizados com doadores vivos ou cadáveres, obedecendo à legislação 
específica brasileira para a regulamentação da disponibilização de órgãos para transplantes, qualquer que seja o tipo de doador ${ }^{9}$.

O reconhecimento dos benefícios do transplante tem levado ao aumento do número de pacientes esperando por um órgão ${ }^{2}$. Entretanto, o suprimento de rim humano para o procedimento é pequeno, o que ocasiona o aumento da fila $\mathrm{e}$ maior tempo de espera 10.

Desigualdades no acesso ao transplante renal têm sido observadas internacionalmente, independentemente do nível socioeconômico do país. Vários fatores estão relacionados à probabilidade de acesso a essa intervenção, como variáveis socioculturais (raça/etnia, sexo, idade, escolaridade, suporte social e crenças pessoais), socioeconômicas (renda e plano privado de saúde), clínicas (presença de comorbidades, tempo em diálise; histocompatibilidade, tipo sanguíneo, acesso a cuidados primários de saúde e tempo de referência ao nefrologista) e geográficas $11,12,13,14,15,16,17,18,19,20$. No Brasil, ainda são raras as pesquisas que avaliem o acesso ao transplante de rim.

Este estudo descreve e analisa os fatores sociodemográficos e clínicos associados ao acesso ao transplante renal (doador vivo e cadáver) para pacientes residentes em Belo Horizonte, Minas Gerais, inscritos na lista de espera no período de 2000 a 2004.

\section{Métodos}

Foi realizado um estudo de coorte não concorrente, no qual cada participante de uma coorte histórica de indivíduos inscritos na lista de espera por um transplante renal, entre 1o de janeiro de 2000 a 31 de dezembro de 2004, foi acompanhado por um período mínimo de 12 meses após a data da inscrição até o seu desfecho clínico (transplante, óbito, exclusão da lista de espera ou permanência na fila ao fim do estudo em 31 de dezembro de 2005). Foram elegíveis para esse estudo os pacientes inscritos na Central Estadual de Transplantes de Minas Gerais (MG Transplantes) residentes em Belo Horizonte, maiores de 18 anos, que não tiveram inscrição para transplante combinado rim/pâncreas, cujos dados estavam completos e consistentes.

A variável resposta foi o tempo decorrido entre a data de inscrição na lista de espera até o transplante, óbito, exclusão da lista ou fim do estudo. As variáveis independentes consideradas foram: sexo; ano e idade de início da diálise; ano e idade de inclusão na lista de espera; tempo em diálise até inclusão na lista de espera; cor da pele; atividade profissional; índice de vulnera- bilidade à saúde (IVS) (Prefeitura Municipal de Belo Horizonte. Estatísticas e mapas. http:// portalpbh.pbh.gov.br/pbh/ecp/comunidade. do?app=estatisticas, acessado em 15/Jan/2011); tipo sanguíneo; causa diagnosticada da doença renal crônica; diagnóstico de diabetes; realização anterior de transplante renal (retransplante); painel reativo de anticorpos (PRA); número de transfusões sanguíneas; número de gravidezes; centro transplantador ao qual o paciente estava vinculado no ato da inscrição; unidade de diálise na qual realizava o tratamento dialítico e vínculo a um centro transplantador.

Os dados sociodemográficos e clínicos dos pacientes que permaneceram ativos na lista de espera até o fim do estudo, em 31 de dezembro de 2005, foram obtidos por meio do programa do Sistema Nacional de Transplantes (SNT) versão 5.0, disponibilizado pelo Ministério da Saúde. Para a coleta de dados dos pacientes que foram transplantados, faleceram ou foram excluídos (desligados) da fila de espera durante o período de acompanhamento, utilizou-se o Formulário para Cadastro de Receptores de Rim arquivado no Complexo MG Transplantes. O desfecho clínico de cada paciente foi confirmado por meio dos relatórios enviados pelas unidades de diálise ao Complexo.

Para estimar o nível socioeconômico dos pacientes, foi utilizado o IVS desenvolvido pela Gerência de Epidemiologia e Informação (GEEPI) da Secretaria Municipal de Saúde de Belo Horizonte. Trata-se de um indicador composto, calculado para cada setor censitário, a partir de diferentes variáveis socioeconômicas e de ambiente (saneamento, habitação, educação, renda e indicadores sociais/de saúde), produzindo um indicador sintético cujo objetivo é analisar as características de grupos populacionais que vivem em determinadas áreas geográficas. Neste estudo, foi utilizada a versão 2003 desse indicador, calculado com base em componentes do Censo Demográfico de 2000 (http://www.ibge.gov.br) e em dados de mortalidade de 2000 a 2002, que classifica as áreas da cidade em quatro categorias homogêneas de risco à saúde: risco baixo, médio, elevado e muito elevado. Dessa forma, para identificar a área de risco da residência dos pacientes inscritos para transplante renal, foram aplicadas técnicas de geoprocessamento, utilizando-se a base geográfica do Município de Belo Horizonte, desenvolvida e gerenciada pela PRODABEL (Empresa de Informática e Informação do Município de Belo Horizonte). Foi possível, assim, relacionar o endereço de residência e o respectivo setor censitário.

Para descrever o perfil sociodemográfico e clínico dos pacientes, foram realizadas análises 
descritivas por meio de proporções, medidas de tendência central e de dispersão. O método de Kaplan-Meier e o teste de log-rank foram usados para estimar as curvas até a ocorrência do transplante e compará-las de acordo com as várias categorias de uma mesma variável. Como não foi possível calcular o tempo mediano até o transplante, pois menos de $50 \%$ da população realizou esse procedimento no decorrer do estudo, foi estimado o percentil 80 da função de sobrevivência por meio do método de Bootstrap 21 .

Considerou-se o tempo de contribuição total dos pacientes, desde a data da inscrição até ao transplante, morte ou fim do estudo, não se levando em conta os períodos nos quais os pacientes estiveram inativos na lista. Aqueles que morreram, foram desligados ou chegaram ao final do estudo foram censurados.

O transplante pode ocorrer a partir da doação de um rim de um doador vivo ou doador cadáver. Assim, neste estudo, ao ser inscrito na lista de espera, o paciente estava em risco de dois eventos de interesse que competiam entre si: ser transplantado por um doador vivo ou cadáver. A ocorrência de um dos eventos exclui a possibilidade de ocorrência do outro, ou seja, os eventos são competitivos. Dessa forma, para estimar as razões de risco (hazard ratios) de ser transplantado, utilizou-se o modelo para riscos competitivos na análise univariada e multivariada. O modelo marginal para riscos competitivos tem a mesma formulação do modelo de Cox clássico estratificado, pois o risco basal é diferente para cada desfecho $\left[\lambda_{1}(t) \neq \lambda_{2}(t)\right]$, sendo estimado o efeito médio das covariáveis para todos os possíveis desfechos 22 .

A partir das variáveis significativas $(p \leq 0,20)$ na análise univariada, procedeu-se à entrada hierarquizada das variáveis, na qual foi ajustado um modelo com as variáveis individuais; em seguida, foram incluídas as variáveis referentes à unidade de diálise e ao centro transplantador e, por último, a variável de contexto do município de residência do paciente (IVS). Para permanência da variável no modelo final, foi considerado um nível de significância de 0,05. Avaliou-se a importância de cada covariável no modelo por meio do teste de Wald. Procedeu-se à análise gráfica dos resíduos de Schoenfeld para a avaliação da suposição de proporcionalidade dos riscos. A qualidade do ajuste do modelo final foi avaliada por análise gráfica dos resíduos de Martingale. As análises estatísticas foram realizadas mediante $o$ software livre R versão 2.8.1 (The R Foundation for Statistical Computing, Viena, Áustria; http:// www.r-project.org).

\section{Resultados}

\section{Perfil dos pacientes inscritos em lista de espera para transplante renal}

Foram identificados 962 pacientes residentes em Belo Horizonte, inscritos na lista de espera por um transplante renal no MG Transplantes, no período de 1o de janeiro de 2000 a 31 de dezembro de 2004. Dessa população, foram excluídos 127 pacientes $(13,2 \%)$, dos quais $41(4,3 \%)$ eram menores de 18 anos; 40 (4,2\%) foram inscritos para transplante combinado rim/pâncreas e 46 $(4,8 \%)$ apresentavam datas incompletas ou inconsistentes.

Dessa forma, foram inscritos 835 pacientes elegíveis para o estudo, perfazendo uma média de 167 inscrições/ano. Desses pacientes, 190 $(22,7 \%)$ foram transplantados, $130(15,6 \%)$ foram a óbito, 129 (15,4\%) foram desligados da lista de espera e $386(46,2 \%)$ permaneciam na fila ao final deste estudo em 31 dedezembro 2005.

Na Tabela 1, pode ser observado que a maioria dos pacientes era do sexo masculino, com início do tratamento dialítico entre os anos de 2001 a 2004 e idade média de 43,6 anos; 95\% deles iniciaram a diálise com menos de 65 anos e foram incluídos na lista de espera por transplante depois de 1,7 ano em média de tratamento. Grande parte era da cor não branca (53,9\%), 81,8\% não exerciam atividade profissional e $76,4 \%$ residiam em áreas de risco médio a elevado à saúde.

Em relação aos aspectos clínicos, verifica-se, na Tabela 2, que os pacientes apresentavam, mais frequentemente, grupo sanguíneo O $(49,4 \%)$; $33,5 \%$ tinham glomerulonefrites como causa principal da doença renal crônica terminal; $18,4 \%$ tinham diabetes e $6,7 \%$ já haviam realizado transplante renal anteriormente ao período do estudo. Grande parte desses pacientes apresentava PRA de 0 a $10 \%(82,8 \%) ; 81,2 \%$ tinham realizado menos de duas transfusões sanguíneas e 52,8\% das mulheres apresentavam menos de duas gravidezes. Quanto ao local de realização das diálises, $50 \%$ faziam o procedimento em quatro unidades principais de diálise, das 11 existentes em Belo Horizonte; a maioria destas não tinha centro transplantador. Quase $40 \%$ dos pacientes se vincularam ao mesmo centro transplantador.

\section{Acesso ao transplante renal}

Em relação ao tempo de espera (Tabela 3), observou-se menor tempo decorrido - quase dois anos - entre a inscrição na lista de espera até o transplante renal para pacientes que iniciaram diálise no período de 2001 a 2004, porém menos de $20 \%$ daqueles que iniciaram tratamento entre 
Tabela 1

Características sociodemográficas dos pacientes inscritos na lista de espera para transplante renal, residentes em Belo Horizonte, Minas Gerais, Brasil, 2000-2004.

\begin{tabular}{|c|c|c|c|c|}
\hline Variáveis & $\begin{array}{l}\text { Total de inscritos } \\
\qquad \begin{array}{c}(\mathrm{n}=835) \\
\mathrm{n}(\%)\end{array}\end{array}$ & $\begin{array}{l}\text { Transplantados } \\
\qquad \begin{array}{c}(n=190) \\
n(\%)\end{array}\end{array}$ & $\begin{array}{l}\text { Não transplantados } \\
\qquad \begin{array}{c}(\mathrm{n}=645) \\
\mathrm{n}(\%)\end{array}\end{array}$ & Valor de p * \\
\hline \multicolumn{5}{|l|}{ Sexo } \\
\hline Masculino & $520(62,3)$ & $119(62,6)$ & $401(62,2)$ & 0,908 \\
\hline Feminino & $315(37,7)$ & $71(37,4)$ & $244(37,8)$ & \\
\hline \multicolumn{5}{|l|}{ Ano de início da diálise } \\
\hline 1980-1995 & $54(6,7)$ & $4(2,3)$ & $50(7,9)$ & 0,029 \\
\hline 1996-2000 & $345(42,8)$ & $79(44,6)$ & $266(42,2)$ & \\
\hline $2001-2004$ & $408(50,6)$ & $94(53,1)$ & $314(49,8)$ & \\
\hline \multicolumn{5}{|l|}{ Idade de início da diálise (anos) } \\
\hline Média \pm DP & $43,6 \pm 12,1$ & $40,7 \pm 12,6$ & $44,4 \pm 11,8$ & 0,001 \\
\hline Mediana & 44,3 & 41,4 & 44,8 & \\
\hline $18-34$ & $192(23,8)$ & $61(34,5)$ & $131(20,8)$ & 0,003 \\
\hline $35-49$ & $343(41,1)$ & $65(36,7)$ & $278(44,1)$ & \\
\hline $50-59$ & $200(24,0)$ & $41(23,2)$ & $159(25,2)$ & \\
\hline $60-64$ & $51(6,1)$ & $8(4,5)$ & $43(6,8)$ & \\
\hline$\geq 65$ & $22(2,6)$ & $2(1,1)$ & $20(3,2)$ & \\
\hline Não se aplica & $3 * \star$ & $3 * \star$ & 0 & \\
\hline \multicolumn{5}{|l|}{ Ano de inclusão na lista } \\
\hline $2000-2002$ & $492(58,9)$ & $124(65,3)$ & $368(57,1)$ & 0,043 \\
\hline $2003-2004$ & $343(41,1)$ & $66(34,7)$ & $277(42,9)$ & \\
\hline Tempo em diálise até inclusão na lista de espera & & & & 0,004 \\
\hline Média \pm DP & $1,7 \pm 2,4$ & $1,2 \pm 1,4$ & $1,9 \pm 2,6$ & \\
\hline Mediana & 0,9 & 0,8 & 0,9 & \\
\hline \multicolumn{5}{|l|}{ Idade de inclusão na lista (anos) } \\
\hline Média \pm DP & $45,4 \pm 12,0$ & $41,9 \pm 12,7$ & $46,3 \pm 11,6$ & 0,000 \\
\hline Mediana & 46,2 & 43 & 46,8 & \\
\hline $18-34$ & $162(19,4)$ & $56(29,5)$ & $106(16,5)$ & 0,001 \\
\hline $35-49$ & $342(41,0)$ & $74(38,9)$ & $268(41,6)$ & \\
\hline $50-59$ & $231(27,7)$ & $43(22,6)$ & $188(29,2)$ & \\
\hline $60-64$ & $61(7,3)$ & $12(6,3)$ & $49(7,6)$ & \\
\hline$\geq 65$ & $38(4,6)$ & $5(2,6)$ & $33(5,1)$ & \\
\hline \multicolumn{5}{|l|}{ Cor } \\
\hline Branca & $329(46,1)$ & $82(50,3)$ & $247(44,9)$ & 0,225 \\
\hline Não branca & $384(53,9)$ & $81(49,7)$ & $303(55,1)$ & \\
\hline \multicolumn{5}{|l|}{ Atividade profissional } \\
\hline Sim & $117(18,2)$ & $40(28,0)$ & $77(15,4)$ & 0,001 \\
\hline Não & $527(81,8)$ & $103(72,0)$ & $424(84,6)$ & \\
\hline \multicolumn{5}{|l|}{ Área de risco à saúde (IVS) } \\
\hline Risco baixo & $197(23,6)$ & $56(29,5)$ & $141(21,9)$ & 0,032 \\
\hline Risco médio, elevado e muito elevado & $636(76,4)$ & $134(70,5)$ & $502(78,1)$ & \\
\hline
\end{tabular}

DP: desvio-padrão; IVS: índice de vulnerabilidade à saúde.

* Valor de p referente à comparação entre os dois grupos, obtido por meio do teste $\chi^{2}$ de Pearson para proporções e teste t de Student para comparação das médias;

** Três pacientes realizaram transplante preemptivo. 
Tabela 2

Características clínicas dos pacientes inscritos na lista de espera para transplante renal, residentes em Belo Horizonte, Minas Gerais, Brasil, $2000-2004$.

\begin{tabular}{|c|c|c|c|c|}
\hline Variáveis & $\begin{array}{l}\text { Total de inscritos } \\
\qquad \begin{array}{c}(n=835) \\
n(\%)\end{array}\end{array}$ & $\begin{array}{l}\text { Transplantados } \\
\qquad(n=190) \\
n(\%)\end{array}$ & $\begin{array}{l}\text { Não transplantados } \\
\qquad \begin{array}{c}(n=645) \\
n(\%)\end{array}\end{array}$ & Valor de $p$ * \\
\hline \multicolumn{5}{|l|}{ Tipo sanguíneo } \\
\hline A & $290(34,8)$ & $91(47,9)$ & $199(30,9)$ & 0,000 \\
\hline$A B$ & $21(2,5)$ & $8(4,2)$ & $13(2,0)$ & \\
\hline B & $111(13,3)$ & $21(11,1)$ & $90(14,0)$ & \\
\hline O & $412(49,4)$ & $70(36,8)$ & $342(53,1)$ & \\
\hline \multicolumn{5}{|c|}{ Causa diagnosticada da doença renal crônica } \\
\hline Glomerulonefrites & $266(33,5)$ & $83(44,6)$ & $183(30,0)$ & 0,000 \\
\hline Hipertensão arterial & $237(29,8)$ & $52(28,0)$ & $185(30,4)$ & \\
\hline Diabetes mellitus & $134(16,9)$ & $13(7,0)$ & $121(19,9)$ & \\
\hline Rins policísticos & $39(4,9)$ & $7(3,8)$ & $32(5,3)$ & \\
\hline Outras ou indeterminada & $119(15,0)$ & $31(16,7)$ & $88(14,4)$ & \\
\hline \multicolumn{5}{|l|}{ Diabetes } \\
\hline $\operatorname{Sim}$ & $144(18,4)$ & $17(9,9)$ & $127(20,8)$ & 0,001 \\
\hline Não & $640(81,6)$ & $155(90,1)$ & $485(79,2)$ & \\
\hline \multicolumn{5}{|l|}{ Retransplante } \\
\hline Sim & $54(6,7)$ & $7(3,7)$ & $47(7,6)$ & 0,061 \\
\hline Não & $750(93,3)$ & $181(96,3)$ & $569(92,4)$ & \\
\hline \multicolumn{5}{|l|}{ PRA (\%) } \\
\hline $0-10$ & $342(82,8)$ & $63(96,9)$ & $279(79,5)$ & 0,003 \\
\hline $11-60$ & $52(12,5)$ & $2(3,1)$ & $50(14,2)$ & \\
\hline$>60$ & $22(5,3)$ & $0(0,0)$ & $22(6,3)$ & \\
\hline \multicolumn{5}{|l|}{ Número de transfusões } \\
\hline $0-2$ & $306(81,2)$ & $121(81,8)$ & $185(80,8)$ & 0,814 \\
\hline$>2$ & $71(18,8)$ & $27(18,2)$ & $44(19,2)$ & \\
\hline \multicolumn{5}{|l|}{ Número de gravidezes } \\
\hline $0-2$ & $65(52,8)$ & $26(53,1)$ & $39(52,7)$ & 0,969 \\
\hline$>2$ & $58(47,2)$ & $23(46,9)$ & $35(47,3)$ & \\
\hline \multicolumn{5}{|l|}{ Centro transplantador } \\
\hline Centro transplantador 1 & $329(39,4)$ & $55(28,9)$ & $274(42,5)$ & 0,007 \\
\hline Centro transplantador 2 & $140(16,8)$ & $43(22,6)$ & $97(15,0)$ & \\
\hline Centro transplantador 3 & $132(15,8)$ & $32(16,8)$ & $100(15,5)$ & \\
\hline Centro transplantador 4 & $75(9,0)$ & $20(10,5)$ & $55(8,5)$ & \\
\hline Centro transplantador 5 & $85(10,2)$ & $17(8,9)$ & $68(10,5)$ & \\
\hline Outros & $74(8,9)$ & $23(12,1)$ & $51(7,9)$ & \\
\hline \multicolumn{5}{|l|}{ Unidade de diálise } \\
\hline Unidade de diálise 1 & $144(17,3)$ & $43(22,9)$ & $101(15,7)$ & 0,072 \\
\hline Unidade de diálise 2 & $108(13,0)$ & $31(16,5)$ & $77(12,0)$ & \\
\hline Unidade de diálise 3 & $101(12,2)$ & $19(10,1)$ & $82(12,8)$ & \\
\hline Unidade de diálise 4 & $65(7,8)$ & $17(9,0)$ & $48(7,5)$ & \\
\hline Unidade de diálise 5 & $53(6,4)$ & $10(5,3)$ & $43(6,7)$ & \\
\hline Unidade de diálise 6 & $91(11,01)$ & $15(8,0)$ & $76(11,7)$ & \\
\hline Outros & $269(32,4)$ & $53(28,2)$ & $216(33,6)$ & \\
\hline \multicolumn{5}{|c|}{ Unidade de diálise em hospital com centro transplantador } \\
\hline Sim & $412(49,3)$ & $89(47,3)$ & $323(50,2)$ & 0,485 \\
\hline Não & $419(50,2)$ & $99(52,7)$ & $320(49,8)$ & \\
\hline
\end{tabular}

* Valor de p referente à comparação entre os dois grupos, obtido por meio do teste $\chi^{2}$ de Pearson para proporções e teste t de Student para comparação das médias. 
Tabela 3

Tempo de acesso (anos) ao transplante renal para 20\% dos pacientes incluídos na lista de espera em Belo Horizonte, Minas Gerais, Brasil, 2000-2004.

\begin{tabular}{|c|c|c|}
\hline Variável & Tempo (anos) & Valor de $p$ * \\
\hline \multicolumn{3}{|l|}{ Sexo } \\
\hline Masculino & 2,077 & 0,996 \\
\hline Feminino & 2,093 & \\
\hline \multicolumn{3}{|l|}{ Ano de início da diálise } \\
\hline 1980-1995 & - & 0,000 \\
\hline $1996-2000$ & 2,858 & \\
\hline $2001-2004$ & 1,811 & \\
\hline \multicolumn{3}{|l|}{ Idade de início da diálise (anos) } \\
\hline 18-34 & 1,244 & 0,008 \\
\hline $35-49$ & 2,973 & \\
\hline $50-64$ & 2,307 & \\
\hline$\geq 65$ & - & \\
\hline \multicolumn{3}{|l|}{ Ano de inclusão na lista } \\
\hline $2000-2002$ & 2,414 & 0,214 \\
\hline 2003-2004 & 1,926 & \\
\hline \multicolumn{3}{|c|}{ Tempo em diálise até inclusão na lista de espera (anos) } \\
\hline$\leq 1$ & 2,027 & 0,032 \\
\hline$>1$ & 2,814 & \\
\hline \multicolumn{3}{|l|}{ Idade de inclusão na lista (anos) } \\
\hline $18-34$ & 1,044 & 0,003 \\
\hline $35-49$ & 2,395 & \\
\hline $50-64$ & 2,589 & \\
\hline$\geq 65$ & - & \\
\hline \multicolumn{3}{|l|}{ Cor } \\
\hline Branca & 1,849 & 0,258 \\
\hline Não branca & 2,573 & \\
\hline \multicolumn{3}{|l|}{ Atividade profissional } \\
\hline Sim & 1,301 & 0,000 \\
\hline Não & 2,814 & \\
\hline \multicolumn{3}{|l|}{ Área de risco à saúde (IVS) } \\
\hline Risco baixo & 1,510 & 0,031 \\
\hline Risco médio, elevado e muito elevado & 2,573 & \\
\hline \multicolumn{3}{|l|}{ Tipo sanguíneo } \\
\hline A & 1,364 & 0,000 \\
\hline$A B$ & 1,822 & \\
\hline B & 3,074 & \\
\hline O & 3,641 & \\
\hline \multicolumn{3}{|l|}{ Causa diagnosticada da doença renal crônica } \\
\hline Glomerulonefrites & 1,575 & 0,004 \\
\hline Hipertensão arterial & 2,307 & \\
\hline Diabetes mellitus & - & \\
\hline Rins policísticos & 2,395 & \\
\hline Outras ou indeterminadas & 2,016 & \\
\hline \multicolumn{3}{|l|}{ Diabetes } \\
\hline Sim & 3,753 & 0,016 \\
\hline Não & 2,068 & \\
\hline
\end{tabular}

(continua) 
Tabela 3 (continuação)

\begin{tabular}{|c|c|c|}
\hline Variável & Tempo (anos) & Valor de $p$ * \\
\hline \multicolumn{3}{|l|}{ Retransplante } \\
\hline $\operatorname{Sim}$ & - & 0,050 \\
\hline Não & 2,016 & \\
\hline \multicolumn{3}{|l|}{ Centro transplantador } \\
\hline Centro transplantador 1 & 2,778 & 0,036 \\
\hline Centro transplantador 2 & 1,296 & \\
\hline Centro transplantador 3 & 2,414 & \\
\hline Centro transplantador 4 & 1,616 & \\
\hline Centro transplantador 5 & 2,504 & \\
\hline Outros & 1,611 & \\
\hline \multicolumn{3}{|l|}{ Unidade de diálise } \\
\hline Unidade de diálise 1 & 1,340 & 0,275 \\
\hline Unidade de diálise 2 & 1,671 & \\
\hline Unidade de diálise 3 & 2,395 & \\
\hline Unidade de diálise 4 & 1,784 & \\
\hline Unidade de diálise 5 & 2,778 & \\
\hline Unidade de diálise 6 & 2,814 & \\
\hline Outros & 2,573 & \\
\hline \multicolumn{3}{|c|}{ Unidade de diálise em hospital com centro transplantador } \\
\hline Sim & 2,573 & 0,214 \\
\hline Não & 1,877 & \\
\hline
\end{tabular}

IVS: índice de vulnerabilidade à saúde.

* Valor de p referente à comparação das curvas de sobrevivência, obtido por meio teste de log-rank.

os anos de 1980 e 1995 conseguiram um enxerto. Os pacientes com idade inferior a 35 anos conseguiram realizar o transplante em menos de dois anos de espera; por sua vez, um percentual inferior a $20 \%$ dos pacientes com mais de 65 anos realizou o procedimento. Observou-se, também, que quanto maior o tempo decorrido da entrada em diálise até inscrição na lista, maior é o tempo de espera até o transplante. Pacientes que exerciam alguma forma de atividade profissional apresentaram metade do tempo de espera para o transplante em relação àqueles sem atividades. Para residentes em áreas de menor risco à saúde, o tempo de acesso foi menor, comparandose com os moradores nas áreas de riscos mais elevados.

Em relação ao perfil clínico, notou-se que esperavam menor tempo pela doação de rim indivíduos do tipo sanguíneo A, com glomerulonefrites como causa de doença renal crônica, sem diabetes, sem histórico de transplante renal anterior, assim como aqueles vinculados ao centro transplantador 2. Ao fim do acompanhamento, observou-se que menos de $20 \%$ dos pacientes diabéticos conseguiram realizar transplante, e os que realizaram esperaram quase quatro anos por um rim.
Na análise multivariada (Tabela 4), verificouse que, a cada aumento de um ano na idade do paciente, a razão de risco de ele ser transplantado diminuiu em $2 \%$ e, a cada um ano a mais em diálise, este risco reduziu em $18 \%$. Os indivíduos que residiam nas três áreas consideradas de maior vulnerabilidade à saúde apresentaram um risco $38 \%$ menor de receberem um rim, enquanto ter tipo sanguíneo B reduziu este em $46 \%$ e O, em 53\%. Pacientes diabéticos apresentaram um risco $43 \%$ menor de receberem um enxerto renal do que aqueles não diabéticos.

Ao se analisar a razão de risco nos modelos marginais para doador vivo e cadáver, separadamente, observou-se que cada ano a mais na idade do paciente diminuiu em $2 \%$, enquanto um ano a mais em diálise reduziu em $23 \%$ o risco de se fazer um transplante intervivos. Pacientes residentes em áreas de maior risco à saúde apresentaram 50\% menor risco de conseguirem um enxerto de um doador vivo; o risco foi $48 \%$ menor para pacientes com tipo sanguíneo $\mathrm{O}$ em relação àqueles do grupo $\mathrm{A}$. Ter diabetes contribuiu para uma redução de $66 \%$ no risco de se conseguir um doador vivo.

Em relação ao acesso ao transplante realizado com órgão de um doador falecido, observou- 
Modelo de riscos competitivos para acesso ao transplante renal de pacientes inscritos na lista de espera em Belo Horizonte, Minas Gerais, Brasil, $2000-2004$

\begin{tabular}{|c|c|c|c|}
\hline Variáveis & $\begin{array}{l}\text { Modelo conjunto } \\
\text { RR (IC95\%) }\end{array}$ & $\begin{array}{l}\text { Doador vivo } \\
\text { RR (IC95\%) }\end{array}$ & $\begin{array}{l}\text { Doador cadáver } \\
\text { RR (IC95\%) }\end{array}$ \\
\hline Idade de inscrição & $0,983(0,968 ; 0,998)$ * & $0,978(0,959 ; 0,998)$ * & $0,989(0,967 ; 1,013)$ \\
\hline Tempo em diálise até inclusão na lista de espera & $0,821(0,734 ; 0,919) * \star$ & $0,771(0,631 ; 0,943)$ * & $0,866(0,762 ; 0,983)$ * \\
\hline \multicolumn{4}{|l|}{ Área de risco à saúde (IVS) } \\
\hline Risco baixo & 1,000 & 1,000 & 1,000 \\
\hline Risco médio, elevado ou muito elevado & $0,624(0,445 ; 0,874)$ ** & $0,499(0,318 ; 0,782) * *$ & $0,819(0,487 ; 1,378)$ \\
\hline \multicolumn{4}{|l|}{ Tipo sanguíneo } \\
\hline A & 1,000 & 1,000 & 1,000 \\
\hline$A B$ & $1,372(0,699 ; 2,694)$ & $1,435(0,556 ; 3,707)$ & $1,316(0,481 ; 3,600)$ \\
\hline $\mathrm{B}$ & $0,539(0,329 ; 0,883)$ * & $0,642(0,327 ; 1,260)$ & $0,453(0,222 ; 0,926)$ * \\
\hline O & $0,468(0,332 ; 0,660) \star \star$ & $0,516(0,319 ; 0,836) * *$ & $0,428(0,263 ; 0,697)$ ** \\
\hline \multicolumn{4}{|l|}{ Diabetes } \\
\hline Não & 1,000 & 1,000 & 1,000 \\
\hline Sim & $0,569(0,336 ; 0,961)$ * & $0,337(0,137 ; 0,830)$ * & $0,830(0,421 ; 1,637)$ \\
\hline
\end{tabular}

IC95\%: intervalo de 95\% de confiança; IVS: índice de vulnerabilidade à saúde; RR: razão de risco.

* $p<0,05$;

** $p<0,01$.

se que apenas o tempo decorrido entre a entrada em diálise e a inclusão na lista de espera e o tipo sanguíneo estiveram associados à realização do procedimento. Cada um ano a mais em diálise reduziu em $13 \%$ o risco de transplante renal. Pacientes com tipo sanguíneo $\mathrm{O}$ apresentaram redução de $57 \%$ e os do tipo B, de $55 \%$, no risco de receberem um enxerto proveniente de um doador cadáver (Tabela 4).

\section{Discussão}

Os resultados sugerem que o tempo de espera pelo transplante renal dos portadores de doença renal crônica terminal em Belo Horizonte é influenciado por variáveis sociodemográficas e clínicas, como idade, tempo em diálise, exercício de uma atividade profissional, área de residência, tipo sanguíneo, presença de diabetes, histórico de transplante renal, causa da doença renal crônica terminal e centro transplantador.

Ao se estimarem as razões de risco para o acesso ao transplante de rim, observaram-se maiores disparidades para o doador vivo, em relação à idade, tempo em diálise, residência segundo área de risco a saúde, tipo sanguíneo e diabetes. Já para doador cadáver, apenas tempo em diálise antes de inclusão na fila de espera e tipo sanguíneo foram variáveis preditoras de acesso ao transplante renal.
É relativamente recente a existência de leis sobre transplantes no Brasil. Do período inicial da realização de transplantes de órgãos até os dias atuais, a atividade transplantadora teve uma evolução considerável em termos de técnicas, resultados, variedade de órgãos transplantados e número de procedimentos realizados 23 . Atualmente, existe legislação específica para a regulamentação da disponibilização de órgãos para transplantes. Todo paciente que precisa da intervenção é inscrito na Lista Única de Receptores do Sistema Nacional de Transplantes do Ministério da Saúde. A fila de transplantes no Sistema Único de Saúde (SUS) para cada órgão ou tecido é única, considerados critérios técnicos, geográficos, de compatibilidade e de urgência específicos para cada órgão. São definidos três tipos de doadores: o cadáver, o vivo parente e o vivo não parente. Os transplantes com doadores vivos podem ser realizados com indivíduos consanguíneos, familiares até o quarto grau (primos). Candidatos à doação não familiares, exceto cônjuges, só podem realizar a doação mediante autorização judicial. Os transplantes com doadores vivos são realizados em caráter eletivo com o doador dísponível ou preferencialmente com o de melhor compatibilidade HLA ${ }^{9}$.

A Política Nacional de Transplantes de Órgãos e Tecidos está fundamentada na legislação (Leis no. 9.434/1997 e 10.211/2001), tendo como 
diretrizes a gratuidade da doação, a beneficência para os receptores e não maleficência para os doadores vivos. Essa política está de acordo com as leis que regem o SUS, sendo baseada nos princípios da universalidade, da integralidade e da igualdade e em consonância com o Art. 198 da Constituição Federal de 1988. Portanto, a equidade nos procedimentos relacionados com transplantes de órgãos no Brasil recebe garantias constitucionais.

Entretanto, o Brasil possui, proporcionalmente, uma das maiores lista de espera para transplantes do mundo, com aumento crescente do tempo de espera. No atual contexto, observa-se que a oferta de órgãos é insuficiente para suprir à demanda de milhares de pacientes que aguardam na fila por um transplante de rim 24 .

Numerosos estudos internacionais têm examinado disparidades no acesso à lista de espera e no transplante, principalmente quando se trata de doador cadáver. Os resultados analisados para a cidade de Belo Horizonte confirmam que indivíduos mais velhos têm maior tempo de espera e menor risco de receber um rim de doador vivo. Em vários países e regiões, foi observado que, das desigualdades demográficas no acesso ao transplante de rim, a idade é uma das maiores barreiras, ou seja, pessoas mais velhas têm menores chances de entrar na lista de espera e de obter um transplante. Dentre as possíveis explicações para essa situação, estão as condições clínicas de um paciente mais velho, a maior presença de comorbidades ou outras condições clínicas que contraindicam a realização do procedimento 14,19 . No caso de nosso estudo, observou-se que a idade é um fator importante para conseguir um doador vivo, corroborando os achados de outras investigações, que também mostram que pacientes mais velhos enfrentam mais dificuldades para conseguirem um doador vivo 25 .

Os pacientes que receberam um rim tanto de doador vivo, quanto doador falecido, apresentaram menor tempo entre o início da diálise e sua inclusão na lista de espera. Maior tempo em diálise antes da realização do transplante tem sido correlacionado com maior morbidade, deterioração da condição clínica do paciente e, consequentemente, menor probabilidade de ser transplantado 14 .

Nossos resultados apontaram menor acesso de pacientes pertencentes ao grupo sanguíneo $\mathrm{O}$ ao transplante de rim, independentemente do tipo de doador. Os tipos sanguíneos $A B$ e $B$ estão associados a maiores taxas de transplante, em oposição a uma menor probabilidade dos pacientes do grupo O 20,26. Nos Estados Unidos, pacientes de tipo sanguíneo $\mathrm{O}$ têm um tempo maior de espera do que aqueles de outros grupos em virtude de eles doarem para receptores não $\mathrm{O} 26$.

Os pacientes diabéticos apresentaram menor risco receberem um rim originário de um doador vivo. O diabetes é considerado forte limitador à realização do transplante, seguido da hipertensão arterial, patologias altamente prevalentes em idosos, o que corrobora a hipótese de que idosos e diabéticos teriam menos chance de serem transplantados. Os pacientes com diabetes apresentam menor taxa de acesso à lista de espera e à realização do transplante, o que pode se dever a comorbidades adicionais e mais severas, limitando sua sobrevida e, provavelmente, o acesso ao transplante renal 8,14.

Em Belo Horizonte, os indivíduos residentes em áreas de maior risco à saúde, isto é, regiões compostas por populações de menor nível sócioeconômico, mostraram maior tempo de espera e menor probabilidade de terem transplantado órgão de doador vivo. São associados a uma menor probabilidade de entrar na lista e obter transplante renal os seguintes fatores socioeconômicos: ter menor status socioeconômico, baixa escolaridade, baixa renda e não ter plano privado de saúde 14,16. As variáveis de condições de pobreza da vizinhança sumarizam as condições socioeconômicas nas quais as pessoas vivem; essas variáveis também detectam gradientes socioeconômicos com ampla variação nos resultados de saúde 15,19.

Os atributos socioeconômicos dos locais nos quais as pessoas vivem podem influenciar no acesso aos serviços de saúde 27 . Nossos resultados mostram a necessidade de melhor entendimento de barreiras aos transplantes em face das características de risco da vizinhança. Menores taxas de transplantes entre pacientes em regiões de maior risco à saúde podem refletir diferenças nessas áreas, no que diz respeito ao acesso ao cuidado médico de boa qualidade, impedimentos para adoção de comportamentos saudáveis e qualidade de moradia.

Nossos resultados não mostraram diferenças sociodemográficas significativas de acesso ao transplante renal por doador cadáver. Diferenças foram observadas apenas para as variáveis clínicas tempo em diálise e tipo sanguíneo. O maior tempo em diálise pode piorar as condições clínicas do paciente, de modo que a realização do transplante seja contraindicada 14; por sua vez, o tipo sanguíneo é considerado um dos requisitos de compatibilidade para realização da intervenção ${ }^{9}$. Portanto, infere-se que o sistema de alocação de órgãos pode ter contribuído para diminuir desigualdades sociodemográficas e socioeconômicas, sendo observadas apenas questões 
clínicas interferindo no acesso ao transplante por doador cadáver.

No Brasil, muitos analistas mostram que várias condições técnicas e políticas adotadas nos últimos anos foram insuficientes para promover o crescimento da atividade transplantadora no país. Em Belo Horizonte, dos transplantes realizados no período estudado, a maioria foi realizada com doador vivo (56,4\%), em oposição aos $10 \%$ realizados na Europa e 30\% nos Estados Unidos 28. Constata-se que, apesar do aumento das taxas de transplante renal, a oferta de órgãos ainda não é suficiente para suprir à demanda. O reflexo disso é a longa fila de espera e a maior porcentagem de doações originadas de doadores vivos 29 .

A utilização de dados administrativos, cujo propósito principal não é a investigação, tem como limitação a presença de dados incompletos ou inconsistentes. Notou-se grande quantidade de dados ausentes (por exemplo, dados referentes à sorologia e HLA), o que limitou a análise dessas variáveis. Devido à ausência de dados econômicos e sociais (raça, escolaridade e renda) fundamentais para avaliar a equidade no acesso ao transplante, foi utilizado o indicador sintético
(IVS) para estimar o nível socioeconômico dos indivíduos; contudo, esse indicador pode subestimar as medidas de efeito dessas variáveis. Além disso, foram utilizados apenas os dados dos pacientes inscritos na lista de transplante, não se considerando o universo dos pacientes submetidos à terapia renal substitutiva. Não se avaliou o acesso à lista de transplante, não sendo possível afirmar, portanto, que o universo de pacientes necessitando de transplante renal esteja representados nesta população.

A importância social e econômica dos transplantes renais, bem como a ausência de estudos que descrevam e analisem os fatores geradores de iniquidades tornam necessária a realização de maiores investigações. Sugere-se então, estudos futuros que focalizem desde a passagem do paciente pela atenção básica até a realização do transplante. Estudos longitudinais prospectivos poderiam ser a melhor opção de desenho para uma avaliação mais completa do acesso ao transplante renal, além de estudos em nível nacional que possam contribuir para uma melhor compreensão dos diversos fatores envolvidos no acesso ao transplante de rim.

\section{Resumo}

O objetivo deste artigo foi analisar os fatores associados ao acesso ao transplante renal com doador vivo e cadáver em Belo Horizonte, Minas Gerais, Brasil. Foi realizado um estudo de coorte não concorrente com pacientes inscritos na lista de espera por transplante renal, entre 2000 e 2004, os quais foram acompanhados até o transplante, óbito, exclusão ou permanência na fila de espera ao fim do estudo em 31 de dezembro de 2005. Utilizou-se o modelo de Cox para riscos competitivos. Dos 835 pacientes, 22,7\% foram transplantados. Apresentaram menor risco de transplante por doador cadáver e vivo os pacientes com maior tempo em diálise e tipo sanguíneo O. Observou-se menor ris- co de transplante intervivos para residentes em uma área de alto risco à saúde e portadores de diabetes. A maior disparidade de acesso foi para transplante renal intervivos, uma vez que não houve diferenças sociodemográficas significativas para transplante por doador cadáver. Pode-se inferir que o sistema de alocação de órgãos contribuiu para amenizar desigualdades sociodemográficas, sendo as questões clínicas mais relevantes no acesso ao transplante com doador cadáver.

Insuficiência Renal Crônica; Transplante de Rim; Acesso aos Serviços de Saúde 


\section{Colaboradores}

E. L. Machado participou da concepção do desenho do estudo e seu planejamento; coleta, análise e interpretação dos dados; redação do artigo; revisão crítica do conteúdo e aprovação da versão final do manuscrito. I. C. Gomes e C. C. César contribuíram na análise estatística dos dados, revisão crítica do conteúdo e aprova- ção da versão final do manuscrito. F. A. Acurcio e M. L. Cherchiglia colaboraram na concepção do desenho do estudo e seu planejamento; revisão crítica do conteúdo e aprovação da versão final do manuscrito. M. C. M. Almeida participou do geoprocessamento dos dados, revisão crítica do conteúdo e aprovação da versão final do manuscrito.

\section{Referências}

1. White SL, Chadban SJ, Jan S, Chapman JR, Cass A How can we achieve global equity in provision renal replacement therapy? Bull World Health Organ 2008; 86:229-37.

2. Mathur AK, Ashby VB, Sands RL, Wolfe RA. Geographic variation in end-stage renal disease incidence and access to deceased donor kidney transplantation. Am J Transplant 2010; 10(4 Pt 2): 1069-80.

3. Coordenação Geral de Alta Complexidade, Departamento de Atenção Especializada, Secretaria de Assistência a Saúde, Ministério da Saúde. Política Nacional de Atenção ao Portador de Doença Renal. Brasília: Ministério da Saúde; 2004.

4. Port FK, Wolfe RA, Mauger EA, Berling DP, Jiang K. Comparison of survival probabilities for dialysis patients vs cadaveric renal transplantation recipients. JAMA 1993; 270:1339-43.
5. Evans RW, Manninen DL, Garrison LP, Hart LG, Blagg CR, Gutman RA, et al. The quality of life of patients with end-stage renal disease. N Engl J Med 1985; 312:553-9.

6. Eggers PW. Effect of transplantation on the Medicare end-stage renal disease program. N Engl J Med 1988; 318:223-9.

7. Medina-Pestana JO, Vaz MLS, Park SI, Garcia VD, Abbud-Filho M, Campos HH. Organ transplantation in Brazil in the year 2002. Transplant Proc 2004; 36:799-801.

8. Cunha CB, Ponce de León AC, Schramm JMA, Carvalho MS, Souza Júnior PRB, Chain R. Tempo até o transplante e sobrevida em pacientes com insuficiência renal crônica no Estado do Rio de Janeiro, Brasil, 1998-2002. Cad Saúde Pública 2007; 23: 805-13. 
9. Manfro CR, Carvalhal GF. Transplante renal. Rev AMRIGS 2003; 47:14-9.

10. Cecka JM. Kidney transplantation from living unrelated donors. Ann Rev Med 2000; 51:393-406.

11. Alexander GC, Ashwini R, Sehgal MD. Barriers to cadaveric renal transplantation among blacks, women, and the poor. JAMA 1998; 280:1148-52.

12. Ayanian JZ, Cleary PD, Weissman JS, Epstein AM. The effect of pacients' preferences on racial differences in access to renal transplantation. N Engl J Med 1999; 341:1661-9.

13. Ellison MD, Edwards LB, Edwards EB, Barker CF. Geographic differences in access to transplantation in the United States. Transplantation 2003; 76:1389-94.

14. Oniscu GC, Schalkwijk AAH, Johnson RJ, Brown H, Forsythe JLR. Equity of access to renal transplant waiting list and renal transplantation in Scotland: cohort study. BMJ 2003; 327:1261.

15. Hall YN, O'Hare AM, Young BA, Boyko EJ, Chertow GM. Neighborhood poverty and kidney transplantation among US Asians and Pacific Islanders with end-stage renal disease. Am J Transplant 2008; 8:2402-9.

16. Kasiske BL, London W, Ellison MD. Race and socioeconomic factors influencing early placement on the kidney transplant waiting list. J Am Soc Nephrol 1998; 9:2142-7.

17. O’Hare AM, Johansen KL, Rodriguez RA. Dialysis and kidney transplantation among patients living in rural areas of the United States. Kidney Int 2006; 69:343-9.

18. Schaeffner ES, Mehta J, Winkelmayer WC. Educational level as a determinant of access to and outcomes after kidney transplantation in the United States. Am J Kidney Dis 2008; 51:811-8.

19. Wolfe RA, Ashby VB, Milford EL, Bloembergen WE, Agodoa LYC, Held PJ, et al. Differences in access to cadaveric renal transplantation in the United States. Am J Kidney Dis 2000; 36:1025-33.
20. Thamer M, Henderson SC, Ray NF, Rinehart CS, Greer JW, Danovitch GM. Unequal access to cadaveric kidney transplantation in California based on insurance status. Health Serv Res 1999; 34:879900.

21. Akritas MG. Bootstrapping the Kaplan-Meier estimator. J Am Stat Assoc 1986; 81:1032-8.

22. Carvalho MS, Andreozzi VL, Codeço CT, Barbosa MTS, Shimakura SE. Análise de sobrevida: teoria e aplicações em saúde. Rio de Janeiro: Editora Fiocruz; 2005.

23. Secretaria de Fiscalização e Avaliação de Programas de Governo, Tribunal de Contas da União. Avaliação do TCU sobre o Programa Doação, Captação e Transplante de Órgãos e Tecidos. Brasília: Tribunal de Contas da União; 2006.

24. Associação Brasileira de Transplantes de Órgãos e Tecidos. Registro Brasileiro de Transplantes. Veículo Oficial da Associação Brasileira de Transplante de Órgãos 2010; Ano XVI, nº 4.

25. Gore JL, Danovitch GM, Litwin MS, Pham PT, Singer JS. Disparities in the utilization of live donor renal transplantation. Am J Transplant 2009; 9: 1124-33.

26. VII. Renal transplantation: access and outcomes. Am J Kidney Dis 1999; 34(2 Suppl 1):S95-101.

27. Williams DR, Collins C. Racial residential segregation: a fundamental cause of racial disparities in health. Public Health Rep 2001; 116:404-16.

28. Oliveira MB, Romão Jr. JE, Zatz R. End-stage renal disease in Brazil: epidemiology, prevention, and treatment. Kidney Int 2005; 68:82-6.

29. Garcia VD. Por uma política de transplantes no Brasil. São Paulo: Office Editora; 2000.

Recebido em 28/Mar/2012

Versão final reapresentada em 16/Jul/2012

Aprovado em 29/Ago/2012 